\title{
Document engineering approaches toward scalable and structured multimedia, web and printable documents
}

\author{
Maria da Graça Pimentel • Dick C. A. Bulterman • \\ Luiz Fernando Gomes Soares
}

Published online: 8 May 2009

C The Author(s) 2009. This article is published with open access at Springerlink.com

\begin{abstract}
Document engineering is the computer science discipline that investigates systems for documents in any form and in all media. As with the relationship between software engineering and software, document engineering is concerned with principles, tools and processes that improve our ability to create, manage, and maintain documents (http://www.documentengineering.org). The ACM Symposium on Document Engineering is an annual meeting of researchers active in document engineering: it is sponsored by ACM by means of the ACM SIGWEB Special Interest Group. In this editorial, we first point to work carried out in the context of document engineering, which are directly related to multimedia tools and applications. We conclude with a summary of the papers presented in this special issue.
\end{abstract}

Keywords Web documents · Print documents · Multimedia documents ·

Scalable documents $\cdot$ Document authoring

Document engineering is the computer science discipline that investigates systems for documents in any form and in all media. As with the relationship between software engineering and software, document engineering is concerned with principles, tools and processes that improve our ability to create, manage, and maintain documents (http://www. documentengineering.org).

The ACM Symposium on Document Engineering is an annual meeting of researchers active in document engineering: it is sponsored by ACM by means of the ACM SIGWEB

\footnotetext{
M. d. G. Pimentel

Instituto de Ciências Matemáticas e de Computação, Universidade de São Paulo, Av. Trabalhador São-carlense, 400-Centro, Caixa Postal: 668, CEP: 13560-970 São Carlos, SP, Brazil e-mail: mgp@icmc.usp.br

D. C. A. Bulterman $(\bowtie)$

CWI: Centrum Wiskunde \& Informatica, Science Park 123, 1098 XG Amsterdam, The Netherlands e-mail: dick.bulterman@cwi.nl

L. F. G. Soares

Departamento de Informática, Pontifícia Universidade Católica do Rio de Janeiro, Rua Marquês de São Vicente 225-Gávea, 22453-900 Rio de Janeiro, RJ, Brazil e-mail: 1fgs@inf.puc-rio.br
} 
Special Interest Group. In this editorial, we first point to work carried out in the context of document engineering, which are directly related to multimedia tools and applications. We conclude with a summary of the papers presented in this special issue.

\section{Document engineering: reviewing a few keywords}

To illustrate how diverse are the contributions the Document Engineering community can bring to researchers interested in Multimedia Tools and Applications, we present in this section a brief discussion associating work reported in the document engineering literature with keywords relevant to multimedia tools and applications: document representations including XML [36], documentation [11], presentation [28], multimedia [2], linking [15], authoring [16] and automatic generation of documents [11, 21,31], and multimedia presentations [18].

Munson [22] divides native document representations into three types: page-oriented (including those obtained from scanned images and those specified in PDF), streamoriented (such as RTF) and tree-structured (including XML), and observes that, within each type, document representations range from the simple to the complex. Document Engineering research relative to document representations to scanned images includes algorithms for image thresholding [33], and relative to PDF includes approaches for the creation of reusable well-structured PDF documents [1]. Arguing that the most important document representation is the Extensible Markup Language (XML), Munson remarks its widespread use on the Web, in business as well as in non-document applications [22].

XML itself has been investigated in several fronts, for instance in terms of extensions that bring notions from functional programming to web authors [34], of prefiltering techniques for efficient XML document processing [17], XML query performance [29, 30] and version control [27]. Extended versions of two of these works are presented in this special issue.

Researchers on multimedia refer to the discussion of techniques for authoring complex XML documents, by Quint and Vattoon [26], both in terms of documentation and presentation. Relative to software documentation, Forward and Lethbridge [10] present the results of a survey of software professionals with the aim of uncovering the perceived relevance of software documentation, as well as the tools and technologies used to maintain, verify and validate such documents.

The research by Lumley et al. [20], discussing a framework for structure, layout and function in documents, has been cited by researches dealing with aspects of documentation and presentation. The recent work by Boyer [3], discussing standards for interactive office documents, has been read by researchers interested in multimedia, presentation and documentation. Researchers interested in those themes also read the recent paper by de Oliveira, J. B [23] which, detailing two algorithms for automatic document page layout, has an extended version presented in this special issue.

An important theme is that of linking, which has been referred to by researchers concerned with XML transformations [24] as well as multimedia [7, 12, 14]. Readers have also demonstrated interested in related work discussing XML databases [29], spam filtering [12] and time-based web applications [19], this last one with an extended version in this issue.

Work related to authoring and to the automatic generation of documents explores several approaches, including transformations [24, 25], structure [5, 6, 13] and layout [20], authoring [9, 25, 35] and browsing [8], and annotations [4, 14].

Regarding multimedia presentations, Cesar et al. [7] have listed a number of use cases in which multimedia content needs to be modified, namely Media Fragmentation, Media 
Enrichment and Media Adaptation. These use cases lead to the identification of a number of requirements with respect to content transformation operations, including the need to provide context-related information, to allow dynamic transformation, to support traceability and search. Moreover, their basic claim is that content transformation activities should be provided as non-destructive operations. The authors argue that these requirements are supported in the literature in terms of contextual information [25], Interactive Television documents [5, 13], end-user enrichments of multimedia content [4, 14], and broadcaster enrichment of television programs [9].

\section{ACM Document Engineering 2008}

DocEng 2008 took place in São Paulo, Brazil, from September 16 to 19, 2008 (http://www. icmc.usp.br/doceng08). DocEng 2008 was the eighth edition of the Symposium, after previous annual meetings at Winnipeg (Canada), Amsterdam (NL), Bristol (UK), MilwaukeeWI (USA), Grenoble (France), McLean-VA (USA), and the first one, in Atlanta-GA (USA).

At DocEng 2008, once again the Document Engineering community has shown itself to be broad and diverse, spanning interests from high-level to low-level document modeling, generation, transformation and production [32]. The DocEng 2008 program committee selected 21 full papers, 19 short papers and six demonstration papers, in one of the most competitive edition of the Symposium to date. All of these contributions were thoroughly refereed, with an average of more than three reviews for the full and short paper submissions, and three reviews for each demonstration paper. A new aspect in the 2008 edition of the Symposium was the introduction of a Best Paper competition, the outcome of which is determined both by a panel of past DocEng program chairs and the audience at the Symposium.

This special issue presents extended versions of six papers selected from DocEng'08, all of them containing significant extended contents relative to the original papers:

- Two papers were selected from the Scalable Documents session, and the extended versions are: "SMIL State: An Architecture and Implementation for Adaptive TimeBased Web Applications", by Jack Jansen and Dick Bulterman, and "Authoring of Scalable Multimedia Documents", by Benoit Pellan and Cyril Concolato.

- One paper was presented in the Structured Documents session, its extended version is called: "Versioning XML-Based Office Documents-An Efficient, FormatIndependent, Merge-Capable Approach", by Sebastian Rönnau and Uwe M. Borghoff.

- One paper was presented in the Document/Image Layout session: the extended version is "Two Algorithms for Automatic Document Page Layout", by João B. S. Oliveira.

- One paper was presented in the Modeling Documents session: Rebeca Schroeder and Ronaldo dos Santos Mello extended version is called "Designing XML Documents from Conceptual Schemas and Workload Information".

- One paper was presented in the Generation and Printing session: "An Exploratory Mapping Strategy for Web-Driven Magazines" is the title extended version of the paper by Fabio Giannetti.

Winners of the best paper award at the Symposium, Jack Jansen and Dick Bulterman's extended paper is called "SMIL State: An Architecture and Implementation for Adaptive Time-Based Web Applications". The paper examines adaptive time-based web applications-interactive presentations where time dictates which parts of the application are 
presented, and that require interactivity and other dynamic adaptation. The paper presents a discussion with respect to technologies which are currently available to create such presentations, as well as their shortcomings. They suggest SMIL State as the mechanism to address the shortcomings identified in current technologies: it can be used to add userdefined state to declarative time-based languages such as SMIL or SVG; as a result, it enables authors to create control flows that are difficult to realize within the temporal containment model of the host languages. The authors also discuss how SMIL State enables easy integration of external components into a web application. SMIL State has been accepted as a part of the standard for SMIL 3.0, the Synchronized Multimedia Integration Language, which became a W3C Recommendation in December 2008.

Benoit Pellan and Cyril Concolato's new paper is called "Authoring of Scalable Multimedia Documents". The authors argue that the adaptation of dynamic and interactive multimedia presentations is essential. They identify the challenge of allowing a multimedia presentation to adapt in all its dimensions: spatially, temporally and interactively, without lessening its attractiveness and still giving the author the control over adapted versions. They also argue that the authoring of adaptable content should not increase the complexity of authoring. Addressing this challenge, they propose the Scalable MSTI model. The paper shows both the properties of the model and how, from an authoring point of view, scalable multimedia documents can be created to address a wide range of defined usage conditions.

Sebastian Rönnau and Uwe M. Borghoff's paper in this issue present an extended version of the paper presented in the Structured Documents session, which is called "Versioning XML-Based Office Documents-An Efficient, Format-Independent, MergeCapable Approach". The authors argue that, although the ability to reliably merge independent updates of a document is a crucial prerequisite to efficient collaboration in office work, merge support for common office document standards such as OpenDocument or OfficeOpenXML is still in its infancy. They present a consistent versioning model for XML documents which includes merge support: they use context-aware fingerprints that identify edit operations and allow the detection of conflicts. The paper details how to extract tracked changes from office documents so they can be mapped in a delta model. The authors also show experimental results indicating the efficiency and reliability of the fingerprinting technique.

João B. S. Oliveira's extended version of the paper originally presented in the Document/Image Layout session is called "Two Algorithms for Automatic Document Page Layout". In the extended version, Oliveira details two approaches to the problem of automatically placing document items on pages. His solutions, which partition the pages into regions where each item is to be placed, work on different input data according to the application. One approach assumes that previously defined rectangular items are to be placed freely on the page, as in a brochure presenting items for sale. The second approach places free-form items on pages divided into columns, as in a newspaper. Both approaches try to preserve the reading order provided by the input and use all available area on the page.

Extending their original paper presented in the Modeling Documents session, Rebeca Schroeder and Ronaldo dos Santos Mello's new paper is called "Designing XML Documents from Conceptual Schemas and Workload Information", and includes a case study for XML databases. The paper tackles the fact that XML schema design has become an important task with the increase of XML-based applications. The authors argue that, although one approach is to consider conceptual schemas as a basis for generating XML documents compliant to consensual information of specific domains, the conversion of conceptual schemas to XML schemas is not a straightforward process; as a result, 
inconvenient design decisions can lead to a poor query processing on XML documents generated. In the paper, Schroeder and Mello present a conversion approach which considers data and query workload estimated for XML applications in order to generate an XML schema from a conceptual schema. The claim is that load information can be used to produce XML schemas which can respond well to the main queries of an XML application. The authors evaluate their approach by means of a case study carried out on a native XML database, and discuss experimental results that demonstrate that the XML schemas generated by their methodology contribute to a better query performance than related approaches.

Fabio Giannetti's original paper was presented in the Generation and Printing session. The extended version, "An Exploratory Mapping Strategy for Web-Driven Magazines" introduces an exploratory mapping strategy to allocate web driven content in a highly graphical publication like a traditional magazine. The work covers two major aspects of the mapping, which enable different levels of flexibility and address different content flowing strategies. The paper also contributes with an evaluation of existing standards with respect to the incorporation of flexible mapping and composition capabilities.

The papers presented in this Special Issue demonstrate the diversity and density of the contributions that the Document Engineering community brings to researchers interested in Multimedia Tools and Applications.

Open Access This article is distributed under the terms of the Creative Commons Attribution Noncommercial License which permits any noncommercial use, distribution, and reproduction in any medium, provided the original author(s) and source are credited.

\section{References}

1. Bagley SR, Brailsford DF, Hardy MR (2003) Creating reusable well-structured PDF as a sequence of component object graphic (COG) elements. In Proceedings of the ACM DocEng'03, 58-67

2. Bertolotti P, Gaggi O (2007) A study on multimedia documents behavior: a notion of equivalence. Multimedia Tools Appl 33(3):301-324. doi:10.1007/s11042-007-0102-2

3. Boyer JM (2008) Interactive office documents: a new face for web 2.0 applications. In Proceedings of the ACM DocEng'08, 8-17

4. Bulterman DC (2003) Using SMIL to encode interactive, peer-level multimedia annotations. In Proceedings of the ACM DocEng'03, 32-41

5. Cesar P, Bulterman DC, Jansen AJ (2006) Benefits of structured multimedia documents in IDTV: the end-user enrichment system. In Proceedings of the ACM DocEng'06, 176-178

6. Cesar P, Vaishnavi I, Kernchen R, Meissner S, Hesselman C, Boussard M, Spedalieri A, Bulterman DC, Gao B (2008) Multimedia adaptation in ubiquitous environments: benefits of structured multimedia documents. In Proceedings of the ACM DocEng'08, 275-284

7. Cesar P, Bulterman DC, Jansen J, da Graca Pimentel M, Barbosa S (2008) Multimedia content transformation: fragmentation, enrichment, and adaptation. In Proceedings of the ACM DocEng'08, 1-2

8. Codocedo V, Astudillo H (2008) No mining, no meaning: relating documents across repositories with ontology-driven information extraction. In Proceedings of the ACM DocEng'08, 110-118

9. de Resende Costa RM, Moreno MF, Rodrigues RF, Soares LF (2006) Live editing of hypermedia documents. In Proceedings of the ACM DocEng'06, 165-172

10. Forward A, Lethbridge TC (2002) The relevance of software documentation, tools and technologies: a survey. In Proceedings of the ACM DocEng'02, 26-33

11. Geyer W, Richter H, Abowd GD (2005) Towards a smarter meeting record-capture and access of meetings revisited. Multimedia Tools Appl 27(3):393-410. doi:10.1007/s11042-005-3815-0 
12. Gómez Hidalgo JM, Bringas GC, Sánz EP, García FC (2006) Content based SMS spam filtering. In Proceedings of the ACM DocEng'06, 107-114

13. Goularte R, Moreira ES, Pimentel MGC (2003) Structuring interactive TV documents. In Proceedings of the ACM DocEng'03, 42-51

14. Goularte R, Cattelan RG, Camacho-Guerrero JA, Inácio VR, Pimentel MGC (2004) Interactive multimedia annotations: enriching and extending content. In Proceedings of the ACM DocEng'04, 84-86

15. Hauglid JO, Heggland J (2008) Savanta - search, analysis, visualisation and navigation of temporal annotations. Multimedia Tools Appl 40(2):183-210. doi:10.1007/s11042-008-0204-5

16. Hsu LH, Liu P, Dawidowsky T (1999) A multimedia authoring-in-the-large environment to support complex product documentation. Multimedia Tools Appl 8(1):11-64. doi:10.1023/A:1009643213925

17. Huang C, Chuang T, Lee H (2005) Prefiltering techniques for efficient XML document processing. In Proceedings of the ACM DocEng'05, 149-158

18. Hwang E, Prabhakaran B (2003) Application-layer protocol for collaborative multimedia presentations. Multimedia Tools Appl 21(2):103-123. doi:10.1023/A:1025502824502

19. Jansen J, Bulterman DC (2008) Enabling adaptive time-based web applications with SMIL state. In Proceedings of the ACM DocEng'08, 18-27

20. Lumley J, Gimson R, Rees O (2005) A framework for structure, layout \& function in documents. In Proceedings of the ACM DocEng'05, 32-41

21. Macedo AA, Baldochi L, Camacho-Guerrero JA, Cattelan RG, Pimentel MD (2008) Automatically linking live experiences captured with a ubiquitous infrastructure. Multimedia Tools Appl 37(2):93-115. doi:10.1007/s11042-007-0131-x

22. Munson E (2009) Document representations. In Özsu MT, Liu L (Eds) Encyclopedia of Database Systems, in press, to appear in 2009, ISBN: 978-0-387-35544-3

23. Oliveira JB (2008) Two algorithms for automatic document page layout. In Proceedings of the ACM DocEng'08, 141-149

24. Pietriga E, Vion-Dury J, Quint V (2001) VXT: a visual approach to XML transformations. In Proceedings of the ACM DocEng'01, 1-10

25. Pimentel MG, Baldochi L, Munson EV (2006) Modeling context Information for capture and access applications. In Proceedings of the ACM DocEng'06, 92-94

26. Quint V, Vatton I (2004) Techniques for authoring complex XML documents. In Proceedings of the ACM DocEng'04, 115-123

27. Rönnau S, Pauli C, Borghoff UM (2008) Merging changes in XML documents using reliable context fingerprints. In Proceedings of the ACM DocEng'08, 52-61

28. Rotard M, Taras C, Ertl T (2008) Tactile web browsing for blind people. Multimedia Tools Appl 37 (1):53-69. doi:10.1007/s11042-007-0170-3

29. Salminen A, Tompa FW (2001) Requirements for XML document database systems. In Proceedings of the ACM DocEng'01, 85-94

30. Schroeder R, Mello R d. (2008) Improving query performance on XML documents: a workload-driven design approach. In Proceedings of the ACM DocEng'08, 177-186

31. Shirmohammadi S, Ding L, Georganas N (2003) An approach for recording multimedia collaborative sessions: design and implementation. Multimedia Tools Appl 19(2):135-154. doi:10.1023/A:1022143111606

32. Simske S, Pellan B, Shilman M (2009) ACM DocEng 2008 recap. SIGWEB Newsl, Winter (Jan. 2009), 1-4. DOI = http://doi.acm.org/10.1145/1457507.1457508

33. Sturgill M, Simske SJ (2008) An optical character recognition approach to qualifying thresholding algorithms. In Proceedings of the ACM DocEng'08, 263-266

34. Thompson S, King PR, Schmitz P (2007) Declarative extensions of XML languages. In Proceedings of the ACM DocEng'07, 89-91

35. Weibel N, Norrie MC, Signer B (2007) A model for mapping between printed and digital document instances. In Proceedings of the ACM DocEng'07, 19-28

36. Westermann U, Klas W (2005) A typed DOM for the management of MPEG-7 media descriptions. Multimedia Tools Appl 27(3):291-322. doi:10.1007/s11042-005-3810-5 


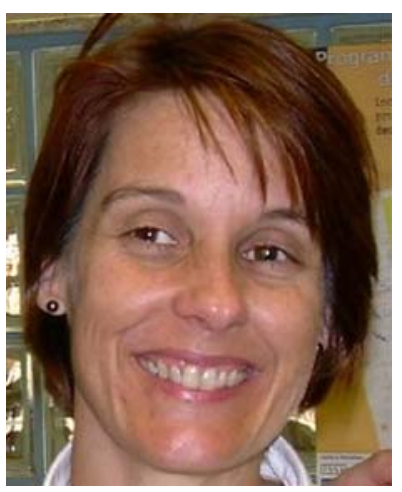

Maria da Graça Pimentel is an Associate Professor of Computer Science Department at the Mathematics and Computer Science Institute of the University of São Paulo-São Carlos, Brazil. She is also the head of Intermedia $\mathrm{Lab}$ at the same institute. Dr. Pimentel received her $\mathrm{PhD}$ in computer science from the University of Kent at Canterbury (UK) in 1994. She has been co-chair of the ACM SIGWEB since 2006. (http://www. icmc.usp. br/ $\sim \mathrm{mgp} /$ )

Pimentel has been active in the Document Engineering community since 2001. She was general chair of the ACM DocEng 2008 Symposium. She is a member of the DocEng Steering Committee since 2008.

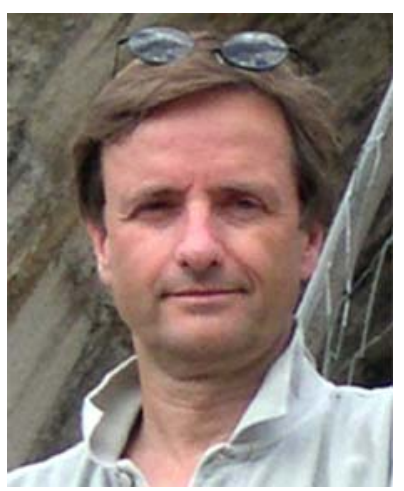

Dick Bulterman is head of distributed multimedia systems research at CWI, the Dutch national center for mathematics and computer science in Amsterdam. He is also a professor of computer science at the VU University in Amsterdam. Dr. Bulterman received his Ph.D. in computer science from Brown University in Providence RI (USA) in 1981. He has been co-chair of the W3C working group on synchronized multimedia since 2007; this group released the SMIL 3.0 Recommendation in late 2008. (http://homepages.cwi.nl/ dcab/)

Bulterman has been active in the Document Engineering community since 2005 . He is past program chair and past general chair of the ACM DocEng Symposium. He is also past chair of ACM Multimedia of and IEEE ISM. 


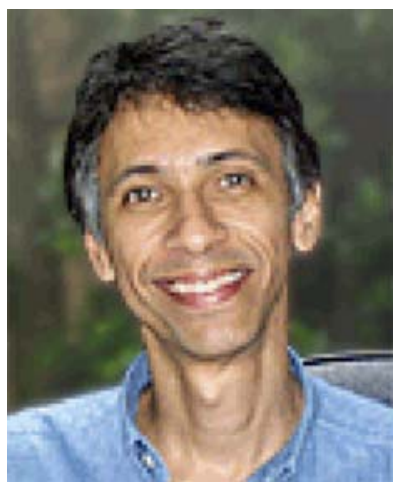

Dr. Luiz Fernando Gomes Soares is a full professor in the Informatics Department at the Catholic University of Rio de Janeiro (PUC-Rio), where since 1990 he heads the TeleMidia Lab. He is a Board Member of the Brazilian Internet Steering Committee and Chair of the Middleware Working Group for the Brazilian Digital TV System. Prior to joining PUC-Rio he was a researcher at the Brazilian Computer Company. Other academic appointments include visiting professorships at École Nationale Superieure de Télécomunications (France), Université Blaise Pascal (France), and Universidad Federico Santa Maria (Chile). He also spent 2 years in the IBM Scientific Center in Rio. Dr. Soares received a D. Sc. in computer science from PUC-Rio (Brazil) in 1983. (http://www2.inf.puc-rio.br/pessoa.lp?id=113\&cxid=professorp)

Soares has been active in the Document Engineering community since 2001. He was Program co-chair of the ACM DocEng 2008 Symposium, and has served in the program committee in all editions of the Symposium. 